\title{
Modelo de minimización de costos asociados al consumo de agua en una industria de refrescos.
}

\author{
Minimization model of cost associated with water consumption in soft drink industry
}

Andrea Russi da Cunha ${ }^{1}$, Urivald Pawlowsky ${ }^{2}$

Recibido: 7/2019

Aceptado 09/2019

\begin{abstract}
Resumen. - En Brasil algunas industrias hidro-intensivas vienen actuando de forma preventiva con respecto a la potencial aprobación de leyes que autoricen el cobro por uso de agua de pozos artesanales. Con el objetivo de conocer posibilidades para minimizar el impacto en los cotos, derivados del consumo de agua en las operaciones de la planta, se evaluaron alternativas para la resolución de un modelo de optimización del uso de recursos hídricos en una planta de refrescos. Específicamente se presenta en este trabajo, el resultado de la aplicación del algoritmo Generalized Bender's Decomposition. El problema se planteó como una superestructura de distribución con el objetivo de minimizar el costo por consumo de agua en las operaciones de la planta. El modelo NLP resultante es del tipo no-convexo. La resolución utilizando descomposición permitió alcanzar sin embargo una respuesta óptima global que derivó en una estructura de distribución de agua con costos menores que la obtenida por la resolución tradicional, que sólo produjo un óptimo local con mayor costo. El problema se resolvió en Gams ${ }^{\circledR}$ con Minos y BDM-LP, obteniendo en menos de 1 segundo los valores que conforman una estructura de distribución con el potencial de reducir los costos por consumo de agua en hasta $17,5 \%$ durante la temporada alta.
\end{abstract}

Palabras clave: Minimización de costos operacionales, redes de distribución, NLP

Summary. - In Brazil, some industries with high consumption of water are developing optimization strategies to prevent or minimize the impact of potential new cost on spring water. The model obtained for the minimization of the water consumption and its operation cost in a soft drink manufacturing facility is NLP and no convex one. This paper shows the results of the application of General Bender's Decomposition algorithm, to a superstructure optimization problem. The use of the GBD algorithm allow nevertheless a global optimal solution which outcome was a water distribution structure with less cost than the one obtained with a traditional NLP solver -local optimum at higher cost. The solver was GAMS $®$ / with BDMLP and Minos @. A global optimum with the values for a structure, that allows a $17 \%$ cost reduction, was obtained in less than one second.

Keywords: Cost Minimization, operational cost reduction, distribution network, NLP, Generalized Bender Decomposition

\footnotetext{
${ }^{1}$ Facultad de Ingeniería Universidad de Montevideo, a.russi@um.edu.uy; ORCID 0000-0002-5767-7659

2 Pos-graduación en Ingeniería Hidráulica y Ambiental. Universidad Federal de Paraná, Brasil.

u.pawlowsky@engquim.ufpr.edu.br
}

Memoria Investigaciones en Ingeniería, 2019. ํ17(1). pp 181-200

https://doi.org/10.36561/ING.17.8

ISSN 2301-1092 • ISSN (en línea) 2301-1106 
1. Introducción. -Las industrias hidro-intensivas, en especial las fabricantes de bebidas ubicadas en Brasil, Argentina, Paraguay o Uruguay, y cuya base de materia prima sea agua, pueden responder a la presión legislativa para la regulación del consumo con estrategias de optimización de la distribución del recurso en planta, [1-4]. En específico, las multas impuestas en la región sur de Brasil sirvieron de estímulo para aplicar diferentes estrategias operativas conducentes a minimizar los costos derivados por el consumo de agua al tiempo que se incrementaba la producción, [5-6].

Los diferentes instrumentos y herramientas de análisis y optimización disponibles en la industria, para la mejora continua de su eficiencia en el consumo de los recursos hídricos, y sus costos asociados, pueden agruparse en dos grandes esquemas generales de abordaje: 1) análisis de redes de consumo de agua y redes de tratamiento de efluentes por separado y 2) análisis integrados de superestructuras de flujos de agua en planta, uso y tratamiento. Varios autores han señalado la preponderancia que han tenido los primeros sobre los segundos, principalmente por la dificultad matemática en la resolución de estos últimos.

Las herramientas utilizadas para la resolución de modelos de optimización de las operaciones tradicionalmente adoptan algoritmos estandarizados y de eficacia de respuesta comprobada. Esta vía práctica podría estar dejando por fuera otros algoritmos de solución con potencial de mejorar aún más la respuesta optimizada, sobre todo en modelos complejos, El potencial de mejora consiste en la posibilidad de garantizar una respuesta óptima global para el sistema modelado, no únicamente óptimo local. Varios autores han propuesto algoritmos para obtener óptimos globales.

El presente trabajo explora la factibilidad de aplicar alguno de ellos a un caso de minimización de costos operativos en la industria de refrigerantes y luego comparar la respuesta obtenida con el uso de los algoritmos estandarizados y evaluar si, más allá de la realidad matemática, existirían razones prácticas para su adopción.

El primer aspecto a considerar fue el diseño de la super-estructura a modelar. Para ello se revisaron modelos aplicados en la minimización costos asociados al consumo de algún recurso costoso (energía, calor y efluentes) [7-13]. Se seleccionó un tipo de acercamiento [13] y se adaptó para ajustar la representatividad de la situación objeto de estudio -industria de refrigerantes con necesidad de optimizar uso de recursos hídricos-. Estas adaptaciones se fundamentaron en los resultados de otras experiencias en la aplicación de dichos modelos; específicamente se revisaron aplicaciones en la industria de refinación petrolera y petroquímica. [12]. Los detalles de la superestructura se presentan en el apartado $\mathrm{N}^{\circ} 1$-Diseño de una superestructura de distribución de agua en planta. - seguidos de la metodología del algoritmo seleccionado para la optimización [8], [14-16]. En la sección de resultados, se les compara para obtener algunas conclusiones y recomendaciones que permitan avanzar en la determinación de instrumentos eficaces para la optimización de procesos industriales, específicamente la minimización de costos asociados al consumo de recursos hídricos.

2. Diseño de una superestructura de distribución de agua en planta. -Alva-Argaez et al. [13] proponen un modelo para optimizar la distribución de efluentes en una refinería, en el cual se indican:

Memoria Investigaciones en Ingeniería, 2019. N17(1). pp 181-200

https://doi.org/10.36561/ING.17.8

ISSN 2301-1092 • ISSN (en línea) 2301-1106 
- Un conjunto de fuentes de agua fresca disponible para satisfacer la demanda, en operaciones que la consumen, definido en términos de flujos límite, concentración de contaminantes y costos

- Un conjunto de operaciones, definidas en términos de la máxima concentración de entrada de ciertos contaminantes claves y la carga de masa del contaminante incrementado dentro de la operación. Un subconjunto "tratamientos", definido en términos de eficiencia en la remoción de contaminantes antes de la entrada a las operaciones o al vertido final.

- Un conjunto de contaminantes claves, significativos para el proceso, que pueden estar presente en las fuentes de agua fresca o ser incorporados en las operaciones que utilizan agua.

La automatización del método recae en la optimización de una superestructura. Todas las posibilidades para la reutilización, regeneración, reciclaje y tratamiento son incluidas en la formulación matemática. En la figura I se presenta el esquema que muestra el modelo general de superestructura para la minimización del consumo de recursos.

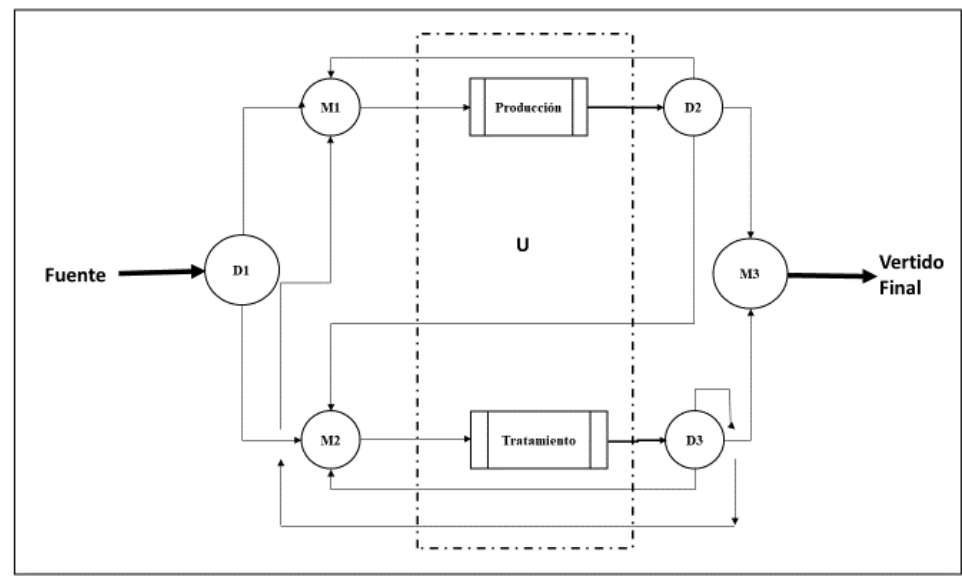

Figura I: Superestrructura general de una topología de red de distribución de recursos hídricos en planta.

- Cada línea de agua que entra en la red se distribuye, en el punto de distribución D1, a todas las unidades i del conjunto $\mathrm{U}$, que procesan o tratan el agua.

- Todas las líneas de efluentes generados en las operaciones se mezclan en un punto final M3 donde se deben alcanzar todas las regulaciones ambientales antes del vertido final.

- $\quad$ Antes de cada operación se consideran los mezcladores M1 y M2, donde los flujos del distribuidor de agua fresca y los flujos de reutilización proveniente de las operaciones, confluyen en un flujo entrante a la operación considerada.

- Después de cada operación se considera un distribuidor, D2 o D3 dependiendo si su origen es procesamiento o tratamiento. De ellos, el flujo se dirige a reutilización en cualquier unidad o al mezclador M3 para vertido.

La función objetivo incluye términos para los costos operacionales de la red -costos de obtención del agua fresca- y costos de capital debido a la instalación de tuberías y habilitación de unidades de tratamiento.

Memoria Investigaciones en Ingeniería, 2019. N17(1). pp 181-200

https://doi.org/10.36561/ING.17.8

ISSN 2301-1092 • ISSN (en línea) 2301-1106 
El modelo matemático incluye balances de masa para cada contaminante en cada mezclador, distribuidor y operación. Para controlar las características estructurales del proyecto, los autores proponen variables binarias asociadas a cada conexión posible. La solución del problema resulta en un consumo mínimo de agua fresca y una topología para la red del proceso. Se identifican las tasas de flujos y concentración de contaminantes en cada línea. Para determinar el costo de capital debido a las tuberías se requieren restricciones relacionando la tasa de flujo con el área transversal de la tubería requerida.

2.1. Componentes de la superestructura aplicada. -Las superestructuras, o modelos de estructuras abiertas, consideran todas las combinaciones tecnológicamente posibles de flujoequipamiento. [8], Cada flujo que entra a la superestructura tiene un costo asociado cuya suma en el total del flujo será minimizada. La respuesta óptima será la estructura de flujoequipamiento cuyo conjunto y valores provee el menor costo total. Si se consideran unidades no instaladas hay que incluir costos de capital y el modelo es del tipo Mixed Integer Nonlinear Problem -MINLP. Con la adaptación propuesta para la aplicación en el caso de estudio de la fábrica de refrescos, sólo se considera capacidad instalada y costos operativos, por ello el modelo a trabaja es del tipo Nonlinear Problem NLP.

- Al respecto de los puntos de mezcla de flujos $M$. El mezclador consiste en una unidad de salida para la unidad correspondiente -sea operación o tratamiento- y un conjunto de corrientes de entrada proveniente de cada uno de los distribuidores. En cada mezclador se establecen balances de masa totales y por contaminante.

- Al respecto de los puntos de distribución de flujos $D$. El distribuidor D consiste en una corriente de entrada proveniente de la fuente o de alguna unidad y un conjunto de corrientes de salida para todos los posibles mezcladores M. Se presenta el balance de masa total y por contaminante. Es necesario forzar la condición para que cada corriente de salida tenga la misma concentración en contaminantes, es decir que la distribución produzca flujos homogéneos y la concentración en la entrada sea la misma que en la salida. Para eso se consideran las concentraciones de salida como constante.

- Al respecto de las unidades de operación o tratamiento $U$. La unidad U consiste en una corriente de entrada proveniente directamente de un mezclador M y una de salida para un distribuidor D. Para cada una de las posibles unidades se presentan los balances de masa totales y por contaminante.

\subsection{Adaptaciones para el caso de estudio de una industria de refrescos. -Alva-Argaez}

et al [13] aplicaron el modelo al proyecto de distribución de agua en una refinería, específicamente en operaciones en las que no había necesidad de considerar incorporación de agua como materia prima al producto, sólo perdidas por evaporación. En tal sentido, para adecuarlo a la realidad de la industria de refrescos, se adecuó el balance de masa en las operaciones de procesamiento considerándose la salida de agua en el producto.

Siguiendo la vía propuesta por Levente et al [12], para el caso de proyectos de aplicación inmediata, que buscan optimizar las eficiencias con pequeños cambios, los costos de inversión en capital pueden no ser considerados y tomar únicamente costos marginales que permitan valorar los flujos que están siendo utilizados en el proceso, y las tuberías o conexiones existentes, aunque no estén operativas.

Memoria Investigaciones en Ingeniería, 2019. N17(1). pp 181-200

https://doi.org/10.36561/ING.17.8

ISSN 2301-1092・ISSN (en línea) 2301-1106 


\section{Metodología:}

3.1. Algoritmos de resolución. -La garantía de una respuesta del tipo "óptimo global" depende en gran medida de la cercanía de la solución inicial del proceso iterativo a la solución óptima. Por ejemplo, con el uso de Minos 5 ®, si el punto inicial estuviere lejos del óptimo entonces la solución final puede converger a un óptimo local con mayor costo.

En el tipo de problema presentado en estas superestructuras, la dificultad en la determinación de la solución surge de la naturaleza de los balances de masa por contaminante en los mezcladores M1 y M2: estos balances originan restricciones que son bilineales, con signo diferente en los flujos y concentración de contaminantes, consecuentemente son restricciones no-convexas y pueden resultar en más de un óptimo local.

Para mejorar la respuesta se optó por un método de descomposición tipo GBD $^{3}$ [16] que continúa siendo aplicado con buenos resultados en optimizaciones de procesos similares [14] [15]. Para esto se clasifica apropiadamente el conjunto de variables y el de las restricciones para descomponer el problema original NLP no convexo en una serie de problemas convexos cuya solución lleve a la solución óptima global. El algoritmo GBD propone seleccionar del conjunto de variables, un subconjunto de complejas y otro de no complejas, en referencia a la relación que tengan con las causas para la no convexidad o la bilinealidad del problema. Luego propone dividir el problema en uno que contenga las restricciones únicamente de variables complejas y otro las de variables complejas y no complejas. De esta división de variables y restricciones se forman el problema primal y el master.

El problema Primal consiste en la función objetivo y el segundo subconjunto de restricciones (aquél con variables complejas y no complejas). Aquí se toman como dato fijo los valores de las variables complejas generadas en el problema Master, o por una solución inicial para comenzar la iteración. La solución del problema Primal se considera el límite superior de la función objetivo original. El problema Master consiste en el primer subconjunto de restricciones (aquél con variables únicamente complejas) y un conjunto de inecuaciones que envuelven la función lagrangeana derivada de la solución del problema primal. Aquí se calculan los valores de las variables complejas y se provee al sistema de un límite inferior de la función objetivo original.

\section{Caso de Estudio: Planta de elaboración de refrescos en el sur de Brasil.}

4.1. Análisis del sistema real. -El sistema se considera como un proceso industrial hidrointensivo, que requiere cierta cantidad de agua -obtenida y procesada a cierto costo-, para elaborar productos. Las fuentes de obtención de agua son la red de distribución industrial estadual y los pozos artesanales dentro de las instalaciones de la fábrica. Los procesos son aquellos que demandan agua como materia prima para la elaboración de productos o generación de vapor, y aquellos que la acondicionan para su consumo interno o para alcanzar requerimientos ambientales de disposición final. Los valores numéricos considerados corresponden a las medias de los períodos de invierno y verano de 3 años consecutivos. No se consideraron las necesidades de agua de los procesos en los cuales ella no sea directamente incorporada en los productos o tratada. Esto excluye procesos de lavado, sanitarios, restauración y comercial. Se consideraron dos factores contaminantes en los flujos de agua:

\footnotetext{
${ }^{3}$ Generalized Bender's Decomposition
}

Memoria Investigaciones en Ingeniería, 2019. № ${ }^{\circ}$ 17(1). pp 181-200

https://doi.org/10.36561/ING.17.8

ISSN 2301-1092・ISSN (en línea) 2301-1106 
alcalinidad y cloro. Estos son los contaminantes controlados en las unidades operacionales: Elaboración de Refrescos y Generación de Vapor, sus concentraciones pueden entonces ser utilizada como valor restrictivo de los flujos entrantes a las operaciones. Los flujos se trabajaron en promedios de dos temporadas de demanda:

- Baja (de junio a septiembre), cuando la planta opera al $45.16 \%$ de su capacidad instalada;

- Alta (de octubre a mayo), cuando la utilización alcanza el 75.3\% de la capacidad instalada.

En la tabla I se presentan los datos de campo con los promedios en L/día. de producción por sabor y empaque en baja y alta temporada.

\begin{tabular}{l|l|l|l|l|l|l|l|l}
\hline & \multicolumn{3}{|c|}{ Baja Temporada 45.16\% capacidad } & \multicolumn{3}{|c}{ Alta Temporada 75.30 \% capacidad } \\
\hline Producto & $\begin{array}{l}\text { Botella } \\
\mathbf{2 9 0} \\
\text { mL }\end{array}$ & $\begin{array}{l}\text { Botella } \\
\mathbf{1 , 5} \mathbf{~ L}\end{array}$ & $\begin{array}{l}\text { Lata } \\
\mathbf{3 5 0} \mathbf{~ m L}\end{array}$ & $\mathbf{T o t a l}$ & $\begin{array}{l}\text { Botella } \\
\mathbf{2 9 0} \\
\text { mL }\end{array}$ & $\begin{array}{l}\text { Botella } \\
\mathbf{1 , 5} \mathbf{L}\end{array}$ & $\begin{array}{l}\text { Lata } \\
\mathbf{3 5 0} \mathbf{~ m L}\end{array}$ & Total \\
\hline Sabor 1 & 52.157 & 92.073 & 145.151 & $\mathbf{2 8 9 . 3 8 2}$ & 64.196 & 145.650 & 220.933 & $\mathbf{4 3 0 . 7 7 9}$ \\
\hline Sabor 2 & 7.306 & 12.168 & 24.616 & $\mathbf{4 4 . 0 9 0}$ & 8.691 & 20.162 & 31.388 & $\mathbf{6 0 . 2 4 0}$ \\
\hline Sabor 3 & 4.016 & 3.707 & 19.181 & $\mathbf{2 6 . 9 0 5}$ & 3.926 & 6.740 & 22.332 & $\mathbf{3 2 . 9 9 8}$ \\
\hline Sabor 4 & 2.250 & 2.671 & 2.640 & $\mathbf{7 . 5 6 1}$ & 2.365 & 6.558 & 16.165 & $\mathbf{2 5 . 0 8 8}$ \\
\hline Sabor 5 & 1339 & 0 & 0 & $\mathbf{1 . 3 3 9}$ & 2.476 & 0 & 0 & $\mathbf{2 . 4 7 6}$ \\
\hline Sabor 6 & 0 & 5.331 & 0 & $\mathbf{5 . 3 3 1}$ & 0 & 0 & 0 & $\mathbf{0}$ \\
\hline Sabor 7 & 2.827 & 4.267 & 6.095 & $\mathbf{1 3 . 1 8 8}$ & 2.822 & 8.891 & 19.886 & $\mathbf{3 1 . 5 9 9}$ \\
\hline Sabor 8 & 0 & 0 & 26.516 & $\mathbf{2 6 . 5 1 6}$ & 0 & 0 & 29.196 & $\mathbf{2 9 . 1 9 6}$ \\
\hline Total & $\mathbf{6 9 . 8 9 5}$ & $\mathbf{1 2 0 . 2 1 8}$ & $\mathbf{2 2 4 . 1 9 9}$ & $\mathbf{4 1 4 . 3 1 2}$ & $\mathbf{8 4 . 4 7 6}$ & $\mathbf{1 8 8 . 0 0 0}$ & $\mathbf{3 3 9 . 9 0 0}$ & $\mathbf{6 1 2 . 3 7 6}$ \\
\hline
\end{tabular}

Tabla I. Producción de refrescos L/día. Fuente: Levantamiento de campo y data histórica

4.2. Modelo Económico. -Se adaptó el modelo propuesto por Alva-Argaez et al [13]. El problema consiste en identificar la estructura óptima de distribución de agua dentro de la planta, de forma que se cumpla el nivel de producción requerido dentro de las especificaciones de calidad del producto y al menor costo posible. El modelo se aplicará sobre la capacidad instalada de la fábrica, considerando el potencial de redistribución y reconexión a un costo de capital despreciable visto que se actúa sobre líneas existentes y/o proyectadas anteriormente. Todos los costos considerados varían proporcionalmente al flujo transportado y el modelo no requiere variables enteras asociadas a la existencia o no de ciertos flujos. El modelo presenta no-linealidad en los balances por componentes en los mezcladores M1 antes de las operaciones -ver Figura I-. Los costos de obtención de agua, presentados en la tabla II, corresponden a:

- Para el agua de la red industrial estadual, el publicado en notificación oficial [17].

- Para el agua de pozo artesanal, el costo de cobranza propuesto por el ante proyecto de Ley para la cobranza de aguas profundas de la bacía hidrográfica Iguazú. [18]. 


\begin{tabular}{c|c}
\hline Fuente & Costo \\
\hline Red Industrial & 1,430 \\
\hline Pozo & $0,201^{4}$ \\
\hline Tabla II Costos de los flujos de agua por tipo de fuente USD/m3
\end{tabular}

En la tabla III se presenta la distribución del consumo actual de agua en las temporadas baja y alta para alcanzar la producción mostrada anteriormente en la tabla II. A seguir en la figura II se muestra la estructura interna de distribución de los flujos a las operaciones de producción y tratamiento.

\begin{tabular}{|c|c|c|}
\hline Fuente & Consumo baja temporada & Consumo alta temporada \\
\hline Red Industrial SANEPAR S & 147,60 & 226,9 \\
\hline Pozo P & 292,62 & 469,60 \\
\hline Total & 440,22 & 696,5 \\
\hline
\end{tabular}

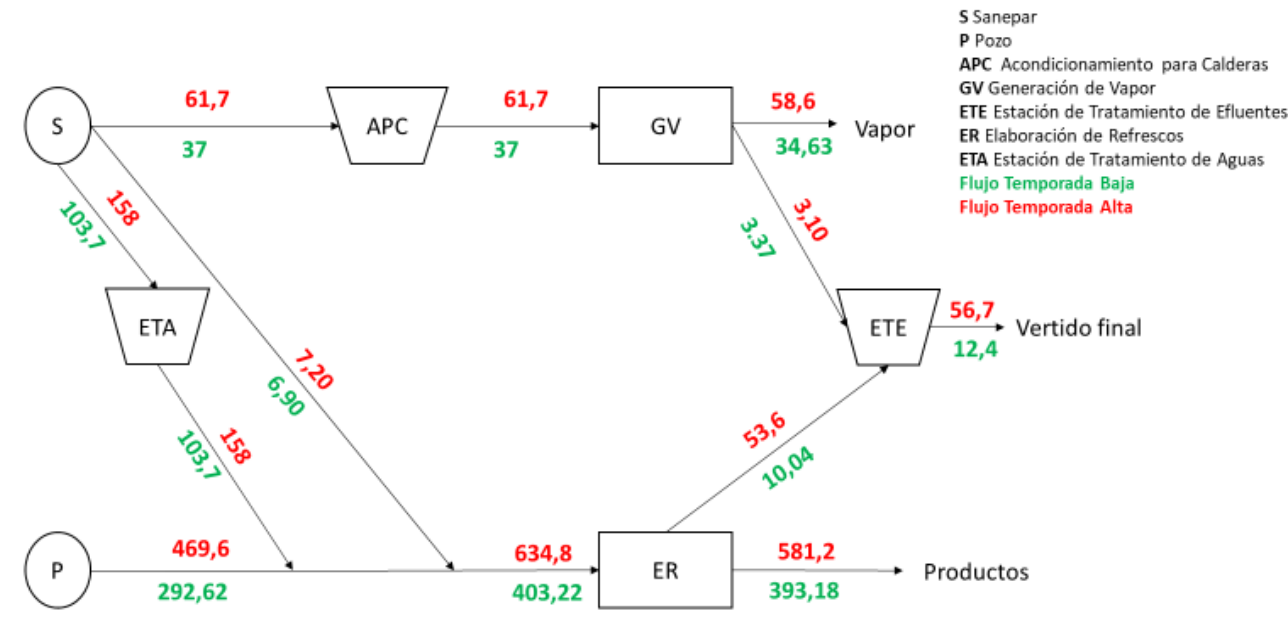

Figura II: Distribución del agua dentro de la planta m3/día por temporada

En estas condiciones de consumo de agua de las distintas fuentes, se define el criterio económico, variable a optimizar, como el costo total por consumo de agua en la planta, bajo la siguiente relación:

Costo Total $=\left(\right.$ Consumo de Agua red industrial $\left(\mathrm{m}^{3} / \mathrm{mes}\right) \times$ Costo unitario del Flujo $\left.\left(\mathrm{USD} / \mathrm{m}^{3}\right)\right)+\left(\right.$ Consumo de Agua Pozo $\left(\mathrm{m}^{3} / \mathrm{mes}\right)$ x Costo unitario del Flujo $\left(\mathrm{USD} / \mathrm{m}^{3}\right)+$ multa por consumo sobre el límite establecido en la licencia de operación.

\footnotetext{
${ }^{4}$ Más USD 1.000 por mes en el que se supere el valor establecido en la licencia de operación. Esta situación es fija en la operación de alta y baja temporada, por lo que en la actualidad mensualmente tienen un costo fijo de USD 1.000,
}

Memoria Investigaciones en Ingeniería, 2019. N17(1). pp 181-200

https://doi.org/10.36561/ING.17.8

ISSN 2301-1092 • ISSN (en línea) 2301-1106 
Estos valores se presentan en la tabla IV para las estructuras de distribución de la figura II.

\begin{tabular}{c|c|c}
\hline Fuente & Costo Temporada Baja & Costo Temporada Alta \\
\hline SANEPAR & $5.487,77$ & $8.436,14$ \\
\hline Pozo & $1.529,23$ & $2.454,13$ \\
\hline Total & $\mathbf{7 . 0 1 7 , 0 0 + 1 . 0 0 0}$ & $\mathbf{1 0 . 8 9 0 , 2 7 + 1 . 0 0 0}$ \\
\hline
\end{tabular}

Tabla IV Costo total actual por consumo de agua por temporada (USD/mes) + multa (USD/mes)

\subsection{Formulación del modelo. -Sean dados:}

a) El conjunto $\mathbf{U}$ de operaciones a ser consideradas en el sistema de agua, separado en dos subconjuntos: El conjunto de todas las operaciones que necesitan agua IO, cada una descrita en términos de la concentración máxima de entrada de cada factor contaminante considerado $\mathrm{W}$ y el conjunto de todas las operaciones que tratan el agua IT, que es descrito por su desempeño en la remoción $\mathrm{R}$ de los factores contaminantes considerados $\mathrm{W}$. Cada operación IO tiene asociado un conjunto de restricciones de flujo F mínimo que entra para garantizar la producción necesaria FP.

b) El conjunto $\mathbf{P}$ de $\mathrm{W}$ factores contaminantes a ser considerado, que están presentes en las fuentes y pueden o no ser removidos en las operaciones de tratamiento IT.

c) El conjunto $\mathbf{A}$ de $\mathbf{J}$ fuentes de agua disponible para alcanzar las exigencias de producción, su composición relativa a los factores contaminantes considerados W. A cada fuente se le asocia un límite superior de consumo de flujo $\mathrm{F}$ en la red de distribución, lo cual corresponde al máximo establecido en la habilitación otorgada a la industria. Se asocia también un costo unitario $\mathrm{C}$ a cada fuente.

Se asume que:

i. Todos los datos para la descripción de las concentraciones máximas de los factores contaminantes W permitidas en las unidades de operación IO están disponibles y son correctos.

ii. El número de operaciones que utilizan IO o que tratan, agua IT,es fijo.

iii. El porcentaje de remoción $\mathrm{R}$ de los factores contaminantes $\mathrm{W}$ es independiente de su concentración en la entrada para una unidad en particular.

iv. Los flujos volumétricos $\mathrm{F}$ no se alteran significativamente con la remoción de los factores $\mathrm{W}$ en las unidades de tratamiento IT.

v. Los valores de las concentraciones de los factores contaminantes $\mathrm{W}$ en los flujos $\mathrm{F}$ tienen mayor significado al ser considerados como parámetro matemático para restringir el flujo de entrada en las unidades que procesan agua, que debe cumplir con requerimientos de calidad de producto. Por otro lado, se asume que los factores contaminantes $\mathrm{W}$ se mezclan homogéneamente.

De los puntos a) a c) y los supuestos i) a v), se obtiene la superestructura específica presentada en la figura III. 


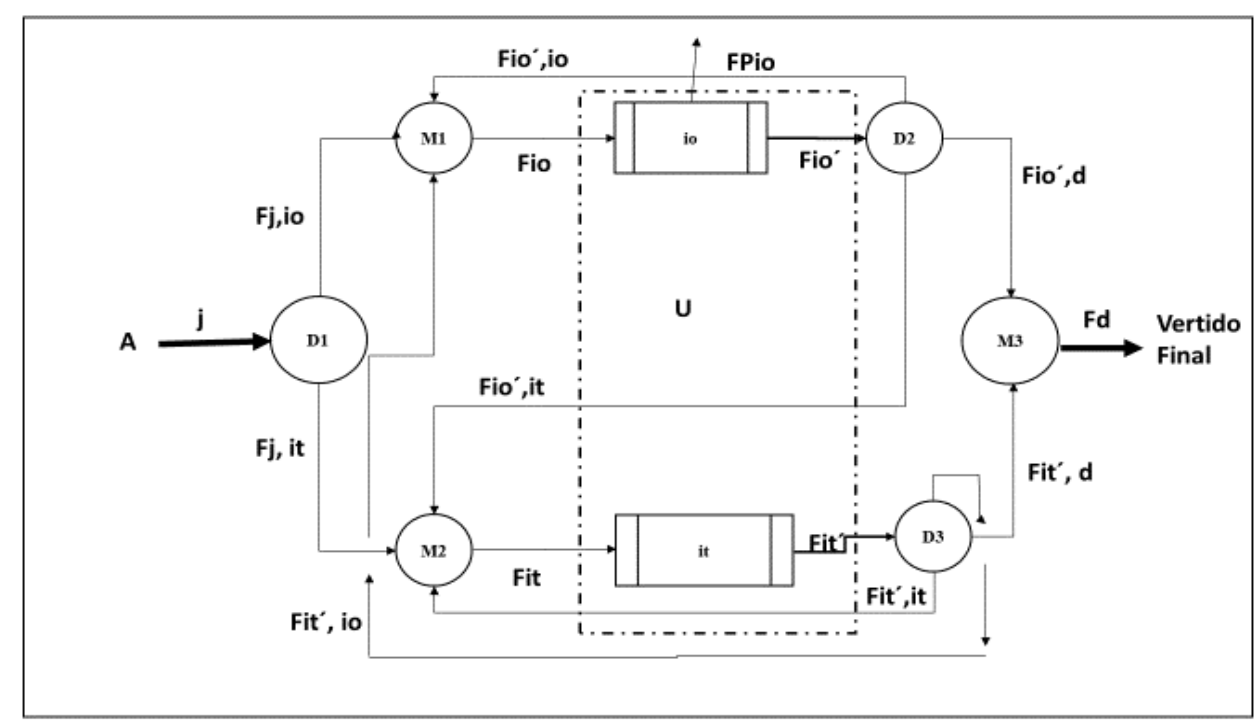

Figura III: Superestructura específica de la red de distribución de recursos hídricos dentro de la Función objetivo: planta

$$
\mathrm{Z}=\min \Sigma_{\mathrm{j}}\left(\mathrm{Costo}_{\mathrm{j}}\right)\left(\mathrm{F}_{\mathrm{j}}\right)
$$

Sujeta a:

Balance de masa en el distribuidor inicial D1

$$
\Sigma_{\text {io }} \mathrm{F}_{\mathrm{j}, \mathrm{io}}+\Sigma_{\mathrm{it}} \mathrm{F}_{\mathrm{j}, \mathrm{it}}-\mathrm{F}_{\mathrm{j}}=0 \quad \forall \mathrm{j} \in \mathrm{A}
$$

Balances de masa en los mezcladores M1 y M2

$$
\begin{aligned}
& \Sigma_{\mathrm{j}} \mathrm{F}_{\mathrm{j}, \mathrm{io}}+\Sigma_{\mathrm{io}} \mathrm{F}_{\mathrm{io},{ }_{\text {,io }}}+\Sigma_{\mathrm{it}}{ }^{\prime} \mathrm{F}_{\mathrm{it}}{ }^{\prime},{ }_{\text {io }}-\mathrm{F}_{\mathrm{io}}=0 \quad \forall \text { io } \in \mathrm{U} \\
& \Sigma_{\mathrm{j}} \mathrm{F}_{\mathrm{j}, \text { it }}+\Sigma_{\mathrm{io}}{ }^{\prime} \mathrm{F}_{\mathrm{io}}{ }^{,}{ }_{\text {it }}+\Sigma_{\mathrm{it}}{ }^{\prime} \mathrm{F}_{\mathrm{it}},{ }_{\mathrm{it}}-\mathrm{F}_{\mathrm{it}}=0 \quad \forall \text { it } \in \mathrm{U}
\end{aligned}
$$

Balance de masa en la unidad que necesita agua

$$
\mathrm{F}_{\text {io }}-\mathrm{F}_{\text {io }} \text { - } \mathrm{FP}_{\text {io }}=0 \quad \forall \text { io,io' } \in \mathrm{U}
$$

Balance de masa en la unidad de tratamiento de agua

$$
\mathrm{F}_{\mathrm{it}}-\mathrm{F}_{\mathrm{it}}{ }^{\prime}=0 \quad \forall \text { it, it' } \in \mathrm{U}
$$

Balances de masa en los distribuidores D2 y D3

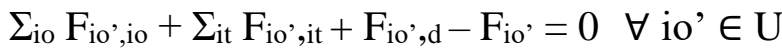

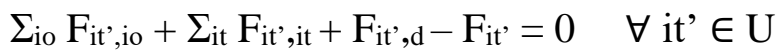

Balance de masa en el mezclador final antes de la descarga del desecho

$$
\Sigma_{\mathrm{io}} \mathrm{F}_{\mathrm{io}, \mathrm{d}}+\Sigma_{\mathrm{it}} \mathrm{F}_{\mathrm{it}}, \mathrm{d}-\mathrm{F}_{\mathrm{d}}=0
$$

Balance de masa por componente en el distribuidor inicial D1

$$
\Sigma_{\text {io }} \mathrm{C}_{\mathrm{w}, \mathrm{j}} \mathrm{F}_{\mathrm{j}, \mathrm{io}}+\Sigma_{\mathrm{it}} \mathrm{C}_{\mathrm{w}, \mathrm{j}} \mathrm{F}_{\mathrm{j}, \mathrm{it}}-\mathrm{C}_{\mathrm{w}, \mathrm{j}} \mathrm{F}_{\mathrm{j}}=0 \quad \forall \mathrm{w} \in \mathrm{P} \text { y } \mathrm{j} \in \mathrm{A}
$$

Balances de masa por componente en los mezcladores M1 y M2

$$
\begin{aligned}
& \Sigma_{\mathrm{j}} \mathrm{C}_{\mathrm{w}, \mathrm{j}} \mathrm{F}_{\mathrm{j}, \mathrm{io}}+\Sigma_{\mathrm{io}} \mathrm{C}_{\mathrm{w}, \text { io }} \mathrm{F}_{\mathrm{io}, \text {,io }}+\Sigma_{\mathrm{it}} \mathrm{C}_{\mathrm{w}, \text { it }} \mathrm{F}_{\mathrm{it} \text { ', io }}-\mathrm{C}_{\mathrm{w}, \text { io }} \mathrm{F}_{\mathrm{io}}=0 \forall \mathrm{w} \in \mathrm{P} \text { y io } \in \mathrm{U} \\
& \Sigma_{\mathrm{j}} \mathrm{C}_{\mathrm{w}, \mathrm{j}} \mathrm{F}_{\mathrm{j}, \mathrm{it}}+\Sigma_{\mathrm{io}} \mathrm{C}_{\mathrm{w}, \mathrm{io}} \mathrm{F}_{\mathrm{io}}{ }^{\prime}, \mathrm{it}+\Sigma_{\mathrm{it}} \mathrm{C}_{\mathrm{w}, \mathrm{it}}{ }^{\prime} \mathrm{F}_{\mathrm{it}}{ }^{\prime},{ }_{\mathrm{it}}-\mathrm{C}_{\mathrm{w}, \mathrm{it}} \mathrm{F}_{\mathrm{it}}=0 \quad \forall \mathrm{w} \in \mathrm{P} \text { y it } \in \mathrm{U}
\end{aligned}
$$

Balance de masa por componente en la unidad que necesita agua

$$
\mathrm{C}_{\mathrm{w}, \text { io }} \mathrm{F}_{\mathrm{io}}-\mathrm{C}_{\mathrm{w} \text {,io }}, \mathrm{F}_{\mathrm{io}},-\mathrm{C}_{\mathrm{w} \text {,io }} \mathrm{Fp}_{\mathrm{io}}=0 \quad \forall \mathrm{w} \in \mathrm{P} \text { y io y io' } \in \mathrm{U}
$$

Balance de masa por componente en unidad de tratamiento de agua con remoción $\mathrm{R}$ de contaminantes 


$$
\mathrm{C}_{\mathrm{w}, \mathrm{it}} \mathrm{F}_{\mathrm{it}}-\mathrm{R}_{\mathrm{w}, \text { it }} \mathrm{C}_{\mathrm{w}, \mathrm{it}} \mathrm{F}_{\mathrm{it}}-\mathrm{C}_{\mathrm{w}, \mathrm{it}} \mathrm{F}_{\mathrm{it}}{ }^{\prime}=0 \quad \forall \mathrm{w} \in \mathrm{P} \text { y it y it' } \in \mathrm{U}
$$

Balances de masa por componente en los distribuidores D2 y D3

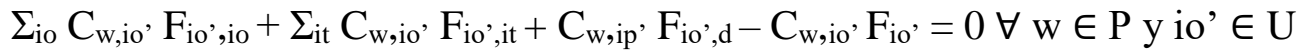

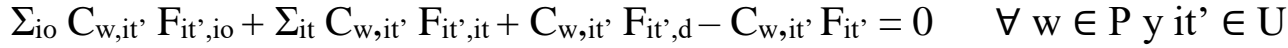

Balance de masa por componente en el mezclador final antes de la descarga del desecho

$$
\Sigma_{\text {io }}, \mathrm{C}_{\mathrm{w}, \mathrm{io}}, \mathrm{F}_{\mathrm{io}, \mathrm{d}}+\Sigma_{\mathrm{it}} \mathrm{C}_{\mathrm{w}, \mathrm{it}} \mathrm{F}_{\mathrm{it}}{ }^{,} \mathrm{d}-\mathrm{C}_{\mathrm{w}, \mathrm{d}} \mathrm{F}_{\mathrm{d}}=0
$$

Restricciones ambientales de consumo de los recursos hídricos

$$
\mathrm{F}_{\mathrm{j}} \leq \operatorname{Limf}_{\mathrm{j}} \quad \forall \mathrm{j} \in \mathrm{A}
$$

Restricciones operacionales de concentración de contaminantes en las unidades que necesitan agua

$$
\mathrm{C}_{\mathrm{w}, \mathrm{io}} \leq \mathrm{Limc}_{\mathrm{w}, \text { io }} \quad \forall \mathrm{w} \in \mathrm{P} \text { y io } \in \mathrm{U}
$$

Restricciones ambientales de concentración de contaminantes en el desecho

\section{Índices}

$$
\mathrm{C}_{\mathrm{w}, \mathrm{d}} \leq \text { Limc }_{\mathrm{w}, \mathrm{d}} \quad \forall \mathrm{w} \in \mathrm{P}
$$

$\mathrm{j}=$ Fuentes de agua disponibles $=\{\mathrm{j} / \mathrm{j} \in($ Red industrial, pozo $)\}$,

io $=$ Operaciones que procesan agua y la utilizan como materia prima $=\{$ io $/$ io $\in$ (Generación de vapor, elaboración de refresco)\},

it $=$ Operaciones que tratan agua removiendo contaminantes $=\{$ it $/$ it $\in($ ETA, APC, ETE $)\}$, io' $=$ Salida de operaciones de procesamiento $=\{$ io' $/$ io' $\in$ (Generación de vapor, elaboración de refresco)\},

it' $^{\prime}$ Salida de las operaciones de tratamiento $=\left\{\right.$ it' $^{\prime}$ it' $\in($ ETA, APC, ETE $\left.)\right\}$,

$\mathrm{d}=$ Desecho final

$\mathrm{w}=$ Factores contaminantes $=\{\mathrm{w} / \mathrm{w} \in($ Alcalinidad, cloro $)\}$.

\section{Variables de decisión}

$\mathrm{F}=$ Flujos de agua

$\mathrm{F}_{\mathrm{j}}=$ Flujo de las fuentes

$\mathrm{F}_{\mathrm{j}, \text { io }}=$ Flujo del distribuidor D1 de la fuente $\mathrm{j}$ al mezclador M1 antes de la operación io

$\mathrm{F}_{\mathrm{j}, \text { it }}=$ Flujo del distribuidor D1 de la fuente $\mathrm{j}$ al mezclador M2 antes de la operación it

$\mathrm{F}_{\mathrm{io}}=$ Flujo que sale del mezclador M1 y entra en la operación io

$\mathrm{F}_{\mathrm{it}}=$ Flujo que sale del mezclador M2 y entra en la operación it

$\mathrm{F}_{\mathrm{io}}{ }^{\prime}=$ Flujo que sale de la operación io y va al distribuidor D2

$\mathrm{F}_{\mathrm{it}}{ }^{\prime}=$ Flujo que sale de la operación it y va al distribuidor D3. Se asume que la cantidad de agua en la remoción de los contaminantes es prácticamente nula y Fit - Fit $^{\prime} \approx 0$

$\mathrm{F}_{\mathrm{io}}$, io = Flujo que sale del distribuidor D2 de la operación io y va para el mezclador M1 antes de la misma operación io, como recirculación.

$\mathrm{F}_{\mathrm{io}}$, it $=$ Flujo que sale del distribuidor D2 de la operación io y va para el mezclador M2 antes de la operación it

$\mathrm{F}_{\mathrm{io}, \mathrm{d}}=$ Flujo que sale del distribuidor D2 de la operación io y va para el mezclador M3 antes del vertido final.

$\mathrm{F}_{\mathrm{it}}$, io $=\mathrm{F}_{\mathrm{io}}$, it $=$ Flujo que sale del distribuidor D3 de la operación it y va para el mezclador M1 antes de operación io

$\mathrm{F}_{\text {it', it }}=$ Flujo que sale del distribuidor D3 de la operación it y va para el mezclador M2 antes de la misma operación it, como recirculación. 
$\mathrm{F}_{\mathrm{it}}, \mathrm{d}=$ Flujo que sale del distribuidor D3 de la operación it y va para el mezclador M3 antes del vertido final.

$\mathrm{F}_{\mathrm{d}}=$ Flujo del vertido Final

$\mathrm{C}_{\mathrm{w}}$, io $=$ Concentración del contaminante $\mathrm{w}$ en el flujo que sale del mezclador M1 y entra en la operación io

$\mathrm{C}_{\mathrm{w}, \text { it }}=$ Concentración del contaminante $\mathrm{w}$ en el flujo que sale del mezclador M2 y entra en la operación it

$\mathrm{C}_{\mathrm{w}, \text { io }}=$ Concentración de contaminante $\mathrm{w}$ que sale de operación io y entra en distribuidor D2

$\mathrm{C}_{\mathrm{w}, \text { it' }}=$ Concentración de contaminante $\mathrm{w}$ que sale de operación it y entra en distribuidor D3

$\mathrm{C}_{\mathrm{w}, \mathrm{d}}=$ Concentración del contaminante $\mathrm{w}$ que sale del distribuidor D3 y va a vertido final

\section{Constantes}

Costoj $=$ Costo de consumo de la fuente de agua fresca

$\mathrm{C}_{\mathrm{w}, \mathrm{j}}=$ Concentración del contaminante $\mathrm{w}$ en la fuente $\mathrm{j}$

$\operatorname{Limf}_{\mathrm{j}}=$ Límite superior del flujo de agua de la fuente $\mathrm{j}$

Limc $_{\mathrm{w}, \text { io }}=$ Límite superior de la concentración del contaminante $\mathrm{w}$ en entrada de operación io

Limc $_{\mathrm{w}, \mathrm{d}}=$ Límite superior de la concentración del factor contaminante $\mathrm{w}$ en el vertido final

$\mathrm{R}_{\mathrm{w}, \text { it }^{5}}{ }^{\mathrm{N}}=$ Fracción de remoción del contaminante $\mathrm{w}$ en la unidad de tratamiento it

$\mathrm{FP}_{\mathrm{io}}=$ Flujo de agua que sale como producto en las operaciones io

Los valores de los costos se presentaron en la tabla IV y a seguir se presentan en la tabla

V los valores límites de las concentraciones de contaminantes medidas en campo, en la tabla VI los límites máximos de consumo permitido por la Agencia Nacional de Regulación de Recursos Hídricos [18], en la tabla VII los valores límite de los contaminantes en los requerimientos de control de calidad de la producción, en la tabla VIII los límites establecidos para el vertido de contaminantes en la regulación ambiental [19], en la tabla IX las fracciones de contaminante removido en it medida en campo como diferencia entre entrada y salida y en la tabla $\mathrm{X}$ los requerimientos de salida de agua constituyente de los productos para alcanzar el parámetro de calidad exigido en la operación.

\begin{tabular}{l|l|l}
\hline & $\begin{array}{l}\text { Red industrial } \\
\text { Sanepar S }\end{array}$ & Pozo P \\
\hline Alcalinidad (mg/m3) & 24.000 & 52.000 \\
\hline Cloro libre (mg/m3) & 6.000 & 0 \\
\hline Tabla $V: C o n c t$ &
\end{tabular}

Tabla V: Concentración de factores contaminantes en el agua de la fuente.

Fuente Datos de campo, media estacional y data histórica de la planta

\begin{tabular}{l|c}
\hline Red Industrial & 775 \\
\hline Pozo & 281 \\
\hline Tabla VI: Flujo máximo de agua de las fuentes (m3/dia) & \\
Fuente: Licencia Operativa de la Empresa.
\end{tabular}

\footnotetext{
${ }^{5}$ El factor de remoción R, no aparece reflejado en el diagrama de la figura III porque no implica flujo de agua, únicamente contaminantes en base seca.
}

Memoria Investigaciones en Ingeniería, 2019. $\mathrm{N}^{\circ} 17(1) . p p$ 181-200

https://doi.org/10.36561/ING.17.8

ISSN 2301-1092•ISSN (en línea) 2301-1106 


\begin{tabular}{l|c|c}
\hline & $\begin{array}{c}\text { Generación de } \\
\text { vapor }\end{array}$ & $\begin{array}{c}\text { Producción de } \\
\text { refrigerante }\end{array}$ \\
\hline Alcalinidad $(\mathrm{mg} / \mathrm{m} 3)$ & 20.000 & 85.000 \\
\hline Cloro libre $(\mathrm{mg} / \mathrm{m} 3)$ & 7.000 & 0 \\
\hline
\end{tabular}

Tabla VII: Concentraciones máximas de factores contaminantes en las operaciones.

Fuente: Manual de Operaciones

\begin{tabular}{l|c}
\hline Factor contaminante & $\begin{array}{c}\text { Concentración } \\
\text { máxima }\end{array}$ \\
\hline Alcalinidad $(\mathrm{mg} / \mathrm{m} 3)$ & 24.000 \\
\hline Cloro libre $(\mathrm{mg} / \mathrm{m} 3)$ & 6.000 \\
\hline
\end{tabular}

Tabla VIII: Concentraciones máximas de factores contaminantes en la descarga. Fuente: Manual de Buenas Prácticas Operaciones con base a límites de descarga a cuerpos de agua en legislación vigente $^{6}$

\begin{tabular}{l|c|c|c}
\hline & $\begin{array}{c}\text { Estación } \\
\text { tratamiento de } \\
\text { agua ETA }\end{array}$ & $\begin{array}{c}\text { Acondicionamient } \\
\text { o para calderas } \\
\text { APC }\end{array}$ & $\begin{array}{c}\text { Estación de } \\
\text { tratamiento de } \\
\text { efluentes ETE }\end{array}$ \\
\hline Alcalinidad & 0,20 & 0,90 & 0,50 \\
\hline Cloro & 1 & 0,32 & 0,99 \\
\hline
\end{tabular}

Tabla IX: Fracción de remoción de factores contaminantes en las operaciones de tratamiento. Fuente: Estimación de campo, diferencia entrada-salida de las operaciones de tratamiento, media estacional

\begin{tabular}{c|c|c}
\hline & $\begin{array}{c}\text { Generación de } \\
\text { vapor }\end{array}$ & $\begin{array}{c}\text { Producción de } \\
\text { refrigerante }\end{array}$ \\
\hline Temporada baja & 34,63 & 393,18 \\
\hline Temporada alta & 58,60 & 581,20 \\
\hline
\end{tabular}

Tabla X: Flujos de agua como producto de las operaciones (m3/día). Fuente: Calculado con base a datos de y parámetros de la producción y el Manual de Operación y control de Calidad de la Planta

El problema modelado entonces en las ecuaciones 1-20 es un problema NLP no convexo, aunque la función objetivo sea lineal y convexa. La no-linealidad aparece en las restricciones 10-17 en los términos bilineales de concentración variable del flujo que sale de los mezcladores multiplicado por los flujos. La no-convexidad se origina en la suma de términos bilineales con signo opuesto.

4.4. Resolución del Modelo. A seguir se presenta la resolución del modelo. siguiendo la propuesta de Floudas, Ciric et al. [14] [15] con base al algoritmo GBD [16] y el solucionador disponible en GAMS $®$ con la idea de procurar un óptimo global. Esta solución se comparará

\footnotetext{
${ }^{6}$ La contaminación en los efluentes no es controlada por la mínima concentración de los mismos en las aguas que son potables.
}

Memoria Investigaciones en Ingeniería, 2019. №17(1). pp 181-200

https://doi.org/10.36561/ING.17.8

ISSN 2301-1092 • ISSN (en línea) 2301-1106 
con la obtenida directamente al introducir el modelo original NLP en la herramienta MINOS también en GAMS [20].

4.4.1. Selección de las variables complejas del problema original (1-20). Las restricciones 10-17 del problema original son bilineales en las concentraciones de contaminantes y flujos de agua. Si se fijan los valores de la variable Flujos, entonces los balances se vuelven lineales en la variable concentración, del mismo modo si se fijan las concentraciones entonces los balances se vuelven lineares en la variable Flujo. Puede entonces seleccionarse un conjunto de variables tal que el sistema quede determinado. Se selecciona la variable Flujo siguiendo prácticas en trabajos anteriores en los que se aplica el algoritmo GBD [21] [22], [23]. De esta forma, una vez eliminadas las redundancias, el problema Primal resultante tiene un óptimo y factible [8], [24]. Para eliminar las redundancias se utilizaron los siguientes ajustes:

- Se definieron variables de holgura:

$\mathrm{HO}_{\mathrm{j}}=$ para el consumo de las fuentes

$\mathrm{H}_{\mathrm{w}}$ io $=$ para la concentración de contaminantes antes de las operaciones

$\mathrm{HD}_{\mathrm{w}, \text { io }}=$ para la concentración de contaminantes en el vertido final

- Se descartó la ecuación (10) ya que la concentración de los contaminantes w en los flujos de las fuentes $\mathrm{Fj}$ es constante y quedaría igual a la ecuación (2) que a su vez se incorporó en la función objetivo. De la misma forma se prescinde de las ecuaciones (15) y (16) de los balances de masa en los distribuidores D2 y D3 luego de cada operación io e it, ya que los flujos quedan determinados en el mezclador inicial M1 de la ecuación (11) y restringido por las ecuaciones (19) y (20). En consecuencia, de las modificaciones anteriores, la función objetivo queda desligada de las restricciones. Para re-asociarla debe ser modificada substituyendo la variable del flujo total de la fuente Fj por la relación de la ecuación (2) correspondiente al balance de masa en el distribuidor inicial D1. Función objetivo original

sujeta a:

$$
\operatorname{Min} \mathrm{Z}=\Sigma_{\mathrm{j}}\left(\mathrm{Costo}_{\mathrm{j}}\right)\left(\mathrm{F}_{\mathrm{j}}\right)
$$

$$
\Sigma_{\text {io }} F_{j, \text { io }}+\Sigma_{\text {it }} F_{\mathrm{j}, \text { it }}-F_{j}=0 \quad \forall \mathrm{j} \in A
$$

Nueva función objetivo

$$
\min \mathrm{Z}=\Sigma_{\mathrm{j}}\left(\text { Costo }_{\mathrm{j}}\right)\left(\Sigma_{\mathrm{io}} \mathrm{F}_{\mathrm{j}, \mathrm{io}}+\Sigma_{\mathrm{it}} \mathrm{F}_{\mathrm{j}, \mathrm{it}}\right)
$$

- Las inecuaciones (18) (19) y (20) se transformaron en ecuaciones agregándose las variables de holgura. Se puede considerar que no interfieren en el hecho de que la solución sea ser única porque no agregan grados de libertad (12 ecuaciones con 24 variables, 12 comunes a las otras restricciones en forma de ecuaciones y 12 nuevas variables de holgura $\mathrm{H}$ ).

$$
\begin{aligned}
& \mathrm{F}_{\mathrm{j}} \leq \operatorname{Limf}_{\mathrm{j}} \quad \forall \mathrm{j} \in \mathrm{A} \rightarrow \quad \Sigma_{\mathrm{io}} \mathrm{F}_{\mathrm{j}, \mathrm{io}}+\Sigma_{\mathrm{it}} \mathrm{F}_{\mathrm{j}, \mathrm{it}}+\mathrm{Hj}-\operatorname{Limf}_{\mathrm{j}}=0 \\
& \mathrm{C}_{\mathrm{w}, \text { io }} \leq \text { Limc }_{\mathrm{w}, \text { io }} \quad \rightarrow \quad \mathrm{C}_{\mathrm{w} \text {,io }}+\mathrm{H}_{\mathrm{w} \text {,io }}-\text { Limc }_{\mathrm{w}, \text { io }}=0 \\
& \mathrm{C}_{\mathrm{w}, \mathrm{d}} \leq \operatorname{Limc}_{\mathrm{w}, \mathrm{d}} \rightarrow \quad \mathrm{C}_{\mathrm{w}, \mathrm{d}}+\mathrm{H}_{\mathrm{w}, \mathrm{d}-\text { Limc }_{\mathrm{w}, \mathrm{d}}=0}
\end{aligned}
$$

Entonces se tiene a seguir la selección de variables complejas definitivas:

$\mathrm{F}_{\mathrm{j}, \text { io }}=$ Flujo del distribuidor D1 de la fuente $\mathrm{j}$ al mezclador M1 antes de la operación io

$\mathrm{F}_{\mathrm{j}, \text { it }}=$ Flujo del distribuidor D1 de la fuente $\mathrm{j}$ al mezclador M2 antes de la operación it

$\mathrm{F}_{\mathrm{io}}=$ Flujo que sale del mezclador M1 y entra en la operación io

$\mathrm{F}_{\mathrm{it}}=$ Flujo que sale del mezclador M2 y entra en la operación it

$\mathrm{F}_{\mathrm{io}}$, io = Flujo que sale del distribuidor D2 de la operación io y va para el mezclador M1 antes de la misma operación io, como recirculación.

Memoria Investigaciones en Ingeniería, 2019. N17(1). pp 181-200

https://doi.org/10.36561/ING.17.8

ISSN 2301-1092・ISSN (en línea) 2301-1106 
$\mathrm{F}_{\mathrm{io}}$, it $=$ Flujo que sale del distribuidor D2 de la operación io y va para el mezclador M2 antes de la operación it

$\mathrm{F}_{\mathrm{io}}, \mathrm{d}=$ Flujo que sale del distribuidor D2 de la operación io y va para el mezclador M3 antes del vertido final.

$\mathrm{F}_{\mathrm{it}}$, io $=\mathrm{F}_{\mathrm{io}}$, it $=$ Flujo que sale del distribuidor D3 de la operación it y va para el mezclador M1 antes de operación io

$F_{i t}$, it = Flujo que sale del distribuidor D3 de la operación it y va para el mezclador M2 antes de la misma operación it, como recirculación.

$\mathrm{F}_{\mathrm{it}}, \mathrm{d}=$ Flujo que sale del distribuidor D3 de la operación it y va para el mezclador M3 antes del vertido final.

$\mathrm{F}_{\mathrm{d}}=$ Flujo del vertido Final

4.4.2. Variables No Complejas del Problema Original. El conjunto de variables no complejas seleccionadas es:

$\mathrm{C}_{\mathrm{w}, \text { io }}=$ Concentración de contaminante $\mathrm{w}$ que sale de mezclador M1 y entra en operación io

$\mathrm{C}_{\mathrm{w}, \text { it }}=$ Concentración del contaminante $\mathrm{w}$ que sale de mezclador M2 y entra en operación it

$\mathrm{C}_{\mathrm{w}}$, io $=$ Concentración del contaminante $\mathrm{w}$ que sale de operación io y entra en distribuidor D2

$\mathrm{C}_{\mathrm{w}, \text { it' }}=$ Concentración del contaminante $\mathrm{w}$ que sale de operación it y entra en el distribuidor D3

$\mathrm{C}_{\mathrm{w}, \mathrm{d}}=$ Concentración del contaminante $\mathrm{w}$ que sale del distribuidor D3 y va a vertido final

\subsubsection{Conjunto de restricciones}

Restricciones que contienen sólo variables complejas

$$
\begin{aligned}
& \Sigma_{\mathrm{j}} \mathrm{F}_{\mathrm{j}, \text { io }}+\Sigma_{\mathrm{io}} \mathrm{F}_{\mathrm{io}} \text {, }{ }_{\text {io }}+\Sigma_{\mathrm{it}}, \mathrm{F}_{\mathrm{it}} \text {, }{ }_{\text {io }}-\mathrm{F}_{\mathrm{io}}=0 \quad \forall \text { io } \in \mathrm{U} \\
& \Sigma_{\mathrm{j}} \mathrm{F}_{\mathrm{j}, \mathrm{it}}+\Sigma_{\mathrm{io}} \text {, } \mathrm{F}_{\mathrm{io}} \text {, it }+\Sigma_{\mathrm{it}}, \mathrm{F}_{\mathrm{it}} \text {, }{ }_{\mathrm{it}}-\mathrm{F}_{\mathrm{it}}=0 \quad \forall \text { it } \in \mathrm{U} \\
& \mathrm{F}_{\text {io }}-\mathrm{F}_{\text {io }},-\mathrm{FP}_{\text {io }}=0 \quad \forall \text { io,io' } \in \mathrm{U} \\
& \mathrm{F}_{\text {it }}-\mathrm{F}_{\text {it' }}=0 \quad \forall \text { it, it' } \in \mathrm{U}
\end{aligned}
$$

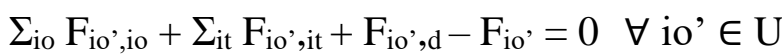

$$
\begin{aligned}
& \Sigma_{\text {io }} F_{\text {it', io }}+\Sigma_{\text {it }} F_{\text {it', }{ }^{\prime}}+F_{\text {it', }}-F_{\text {it' }}=0 \quad \forall \text { it' } \in \mathrm{U} \\
& \Sigma_{\mathrm{io}} \mathrm{F}_{\mathrm{io}, \mathrm{d}}+\Sigma_{\mathrm{it}} \mathrm{F}_{\mathrm{it}}, \mathrm{d}-\mathrm{F}_{\mathrm{d}}=0 \\
& \Sigma_{\text {io }} \mathrm{F}_{\mathrm{j}, \mathrm{io}}+\Sigma_{\mathrm{it}} \mathrm{F}_{\mathrm{j}, \mathrm{it}}+\mathrm{Hj}-\mathrm{Limf}_{\mathrm{j}}=0 \quad \forall \mathrm{j} \in \mathrm{A}
\end{aligned}
$$

Restricciones con variables complejas y no complejas

$$
\begin{aligned}
& \Sigma_{\mathrm{j}} \mathrm{C}_{\mathrm{w}, \mathrm{j}} \mathrm{F}_{\mathrm{j}, \text { io }}+\Sigma_{\mathrm{io}} \mathrm{C}_{\mathrm{w}, \text { io }} \mathrm{F}_{\mathrm{io}, \text {,io }}+\Sigma_{\mathrm{it}} \mathrm{C}_{\mathrm{w}, \text { it }} \mathrm{F}_{\mathrm{it}, \text {,io }}-\mathrm{C}_{\mathrm{w}, \text { io }} \mathrm{F}_{\mathrm{io}}=0 \quad \forall \mathrm{w} \in \mathrm{P} \text { y io } \in \mathrm{U} \\
& \Sigma_{\mathrm{j}} \mathrm{C}_{\mathrm{w}, \mathrm{j}} \mathrm{F}_{\mathrm{j}, \mathrm{it}}+\Sigma_{\mathrm{io}} \mathrm{C}_{\mathrm{w}, \mathrm{io}}, \mathrm{F}_{\mathrm{io},{ }_{\text {,it }}}+\Sigma_{\mathrm{it}} \mathrm{C}_{\mathrm{w}, \mathrm{it}} \mathrm{F}_{\mathrm{it}, \text {,it }}-\mathrm{C}_{\mathrm{w}, \mathrm{it}} \mathrm{F}_{\mathrm{it}}=0 \quad \forall \mathrm{w} \in \mathrm{P} \text { y it } \in \mathrm{U} \\
& \mathrm{C}_{\mathrm{w}, \text { io }} \mathrm{F}_{\mathrm{io}}-\mathrm{C}_{\mathrm{w}, \text { io }}, \mathrm{F}_{\mathrm{io}},-\mathrm{C}_{\mathrm{w}, \text { io }} \mathrm{Fp}_{\text {io }}=0 \quad \forall \mathrm{w} \in \mathrm{P} \text { y io y io' } \in \mathrm{U} \\
& \mathrm{C}_{\mathrm{w}, \text { it }} \mathrm{F}_{\mathrm{it}}-\mathrm{R}_{\mathrm{w}, \text { it }} \mathrm{C}_{\mathrm{w}, \mathrm{it}} \mathrm{F}_{\mathrm{it}}-\mathrm{C}_{\mathrm{w}, \mathrm{it}}{ }^{\prime} \mathrm{F}_{\mathrm{it}}{ }^{\prime}=0 \forall \mathrm{w} \in \mathrm{P} \text { y it y it' } \in \mathrm{U} \\
& \Sigma_{\mathrm{io}} \mathrm{C}_{\mathrm{w}, \mathrm{io}}, \mathrm{F}_{\mathrm{io}, \mathrm{d}}+\Sigma_{\mathrm{it}} \mathrm{C}_{\mathrm{w}, \mathrm{it}} \mathrm{F}_{\mathrm{it}, \mathrm{d}}-\mathrm{C}_{\mathrm{w}, \mathrm{d}} \mathrm{F}_{\mathrm{d}}=0 \\
& \mathrm{C}_{\mathrm{w}, \text { io }}+\mathrm{Hw} \text {,io }-\mathrm{Limc}_{\mathrm{w}, \text { io }}=0 \quad \forall \mathrm{w} \in \mathrm{P} \text { y io } \in \mathrm{U} \\
& \mathrm{C}_{\mathrm{w}, \mathrm{d}}+\mathrm{Hw}, \mathrm{d}-\text { Limc }_{\mathrm{w}, \mathrm{d}}=0 \quad \forall \mathrm{w} \in \mathrm{P}
\end{aligned}
$$

4.4.4. Problema Primal P1. Siguiendo el algoritmo propuesto por Floudas et al. [15], [16], el problema primal se construye con la función objetivo y el conjunto de restricciones conteniendo variables complejas y no complejas. Como se escogió la variable flujo como la

Memoria Investigaciones en Ingeniería, 2019. №17(1). pp 181-200

https://doi.org/10.36561/ING.17.8

ISSN 2301-1092 • ISSN (en línea) 2301-1106 
compleja, entonces al fijarse sus valores iniciales en este problema serán calculados los valores de las variables no complejas -concentraciones- y los multiplicadores de Lagrange para cada restricción. A continuación, se presenta el problema primal, resaltando en negrita los valores conocido por levantamiento en campo (solución inicial) o por resultado del problema Master de las iteraciones siguientes.

Función objetivo sujeta a

$$
\min Z=\Sigma_{\mathrm{j}}\left(\mathbf{C o s t o}_{\mathbf{j}}\right)\left(\Sigma_{\mathrm{io}} \mathbf{F}_{\mathbf{j}, \mathbf{i o}}+\Sigma_{\mathrm{it}} \mathbf{F}_{\mathbf{j}, \mathbf{i t}}\right)
$$

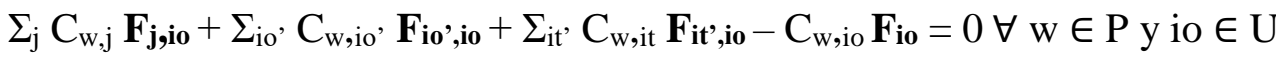

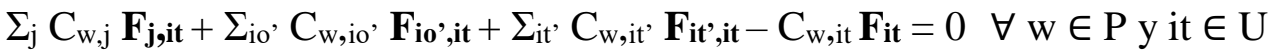

$$
\begin{aligned}
& \mathrm{C}_{\mathrm{w}, \text { io }} \mathbf{F}_{\mathrm{io}}-\mathrm{C}_{\mathrm{w}, \text { io }}, \mathbf{F}_{\mathrm{io}},-\mathrm{C}_{\mathrm{w}, \text { io }} \mathbf{F p}_{\mathrm{io}}=0 \quad \forall \mathrm{w} \in \mathrm{P} \text { y io y io' } \in \mathrm{U} \\
& \mathrm{C}_{\mathrm{w}, \text { it }} \mathbf{F}_{\text {it }}-\mathrm{R}_{\mathrm{w}, \text { it }} \mathrm{C}_{\mathrm{w}, \mathrm{it}} \mathbf{F}_{\text {it }}-\mathrm{C}_{\mathrm{w}, \text { it }} \mathbf{F}_{\text {it' }}=0 \forall \mathrm{w} \in \mathrm{P} \text { y it y it' } \in \mathrm{U} \\
& \Sigma_{\mathrm{io}}, \mathbf{C}_{\mathrm{w}, \mathrm{io}}, \mathbf{F}_{\mathbf{i o}}, \mathbf{d}+\Sigma_{\mathrm{it}} \mathbf{C}_{\mathrm{w}, \mathrm{it}^{\prime}} \mathbf{F}_{\mathbf{i t}}, \mathbf{d}-\mathbf{C}_{\mathrm{w}, \mathrm{d}} \mathbf{F}_{\mathbf{d}}=0 \\
& \mathrm{C}_{\mathrm{w}, \text { io }}+\mathrm{Hw} \text {,io }- \text { Limc }_{\mathrm{w}, \text { io }}=0 \quad \forall \mathrm{w} \in \mathrm{P} \text { y io } \in \mathrm{U} \\
& \mathrm{C}_{\mathrm{w}, \mathrm{d}}+\mathrm{Hw}, \mathrm{d}-\mathbf{L i m c}_{\mathrm{w}, \mathrm{d}}=0 \quad \forall \mathrm{w} \in \mathrm{P}
\end{aligned}
$$

El problema primal tiene las siguientes características:

- Un bloque de 56 ecuaciones descritas en el intervalo (22) -(28)

- 56 variables

- La función objetivo se describe en la ecuación (21), su valor es fijo y sin grados de libertad.

- La solución para las variables complejas es única y óptima.

4.4.5. Problema Master P2. El problema Master P2 se construye con el conjunto de restricciones conteniendo sólo variables complejas y un conjunto de inecuaciones envolviendo la función lagrangeana derivada de la solución del problema Primal. En este sub-problema las variables complejas son calculadas y la solución fija un límite inferior para la función objetivo original. En negrita los valores constantes conocidos,

Función objetivo;

$\min \mathrm{Z}=\mu$

Sujeta a:

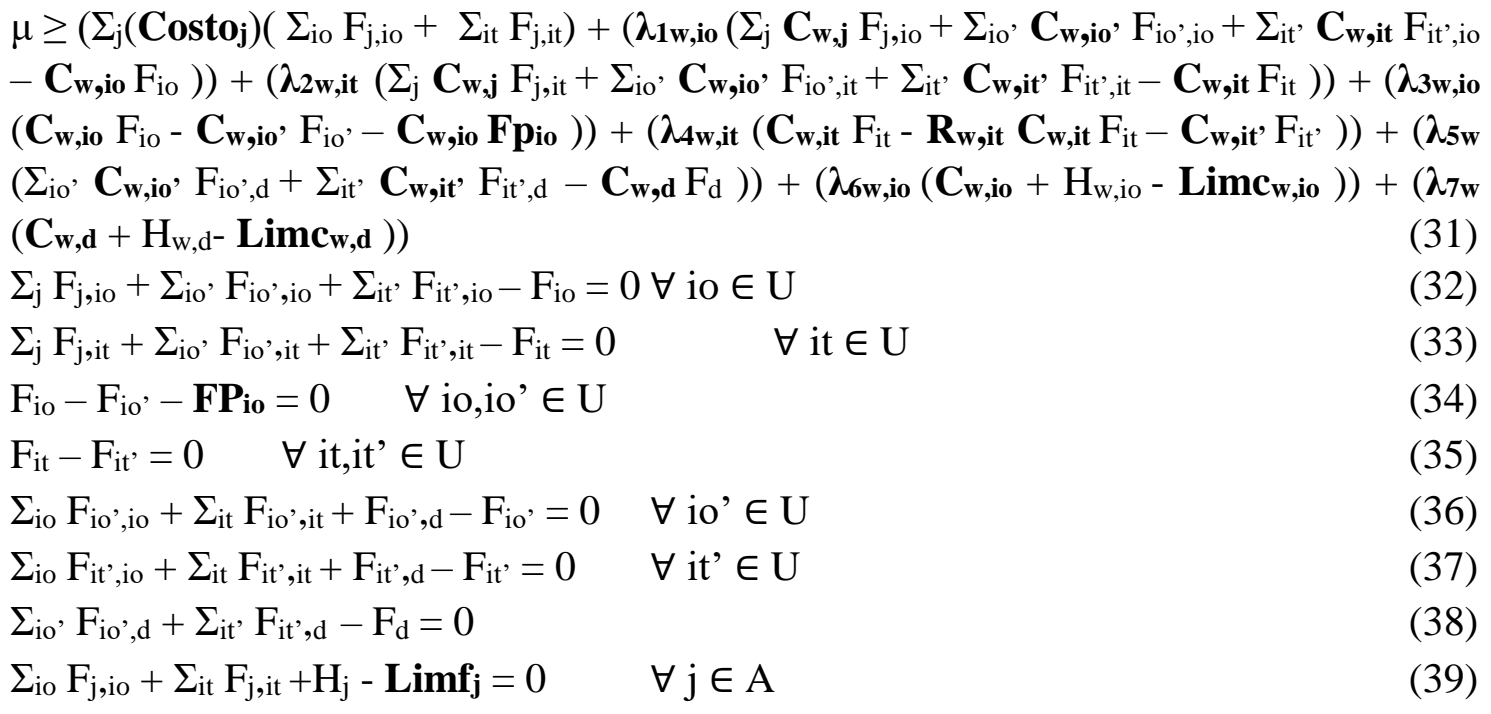

Memoria Investigaciones en Ingeniería, 2019. N17(1). pp 181-200

https://doi.org/10.36561/ING.17.8

ISSN 2301-1092 • ISSN (en línea) 2301-1106 
5. Resultados. Se introducen los modelos obtenidos con el algoritmo GBD en el solucionador BDM-LP de GAMS para comenzar las iteraciones. La solución inicial $\mathrm{K}=0$ corresponde a los valores de los flujos actuales de la planta en temporada alta y baja. Estos datos se presentaron en la tabla III, Figura II y la Tabla IV que muestra los valores de la función objetivo para la solución inicial. El procesamiento se hizo en Windows 10, un procesador Intel Core i7-5500 2.40 GHz CPU y memoria RAM de 8 GB.

En la tabla XI y en la Figura IV se presentan los resultados y la estructura de distribución obtenida para los datos de temporada baja, y en la Tabla XII y Figura V los correspondientes a la temporada alta.

\begin{tabular}{l|c|c}
\hline Interacción K & $\begin{array}{c}\text { Sub-problema } \\
\text { Primal }\end{array}$ & $\begin{array}{c}\text { Sub-problema } \\
\text { Master }\end{array}$ \\
\hline $\mathrm{K}=1$ & 308,41 & 306,22 \\
\hline $\mathrm{K}=2$ & 306,22 & 306,22 \\
\hline Óptimo global & \multicolumn{2}{|c|}{306,22000} \\
\hline
\end{tabular}

Tabla XI: Resultado mínimo de la función objetivo en temporada baja (USD/día)

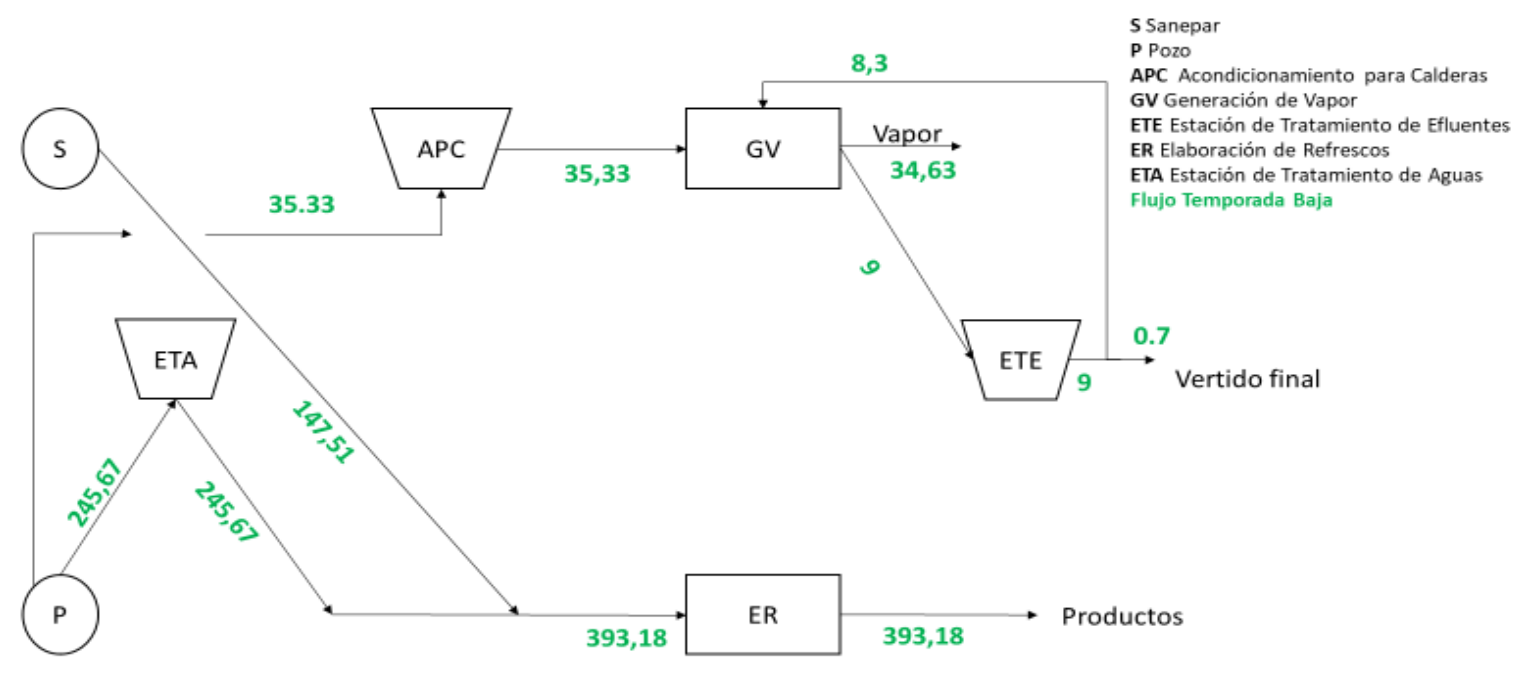

Flujo $\mathrm{j}=$ red industrial $=147,510 \mathrm{m3} / \mathrm{dia}$

$\mathrm{j}=$ poso artesanal $=281 \mathrm{~m} 3 /$ día

Figura IV: Distribución de agua dentro de la planta (m3/día). Temporada Baja. Fuente: Resultado Iteración $K=2$

\begin{tabular}{l|c|c}
\hline Interacción K & Sub-problema Primal & Sub-problema Master \\
\hline $\mathrm{K}=1$ & 478,53 & 338,37 \\
\hline $\mathrm{K}=2$ & 338,37 & 338,37 \\
\hline Óptimo global & \multicolumn{2}{|c}{338,37000} \\
\hline
\end{tabular}

Tabla XII: Resultado mínimo de la función objetivo en temporada alta (USD/día)

Memoria Investigaciones en Ingeniería, 2019. $\mathrm{N}^{\circ} 17(1)$. pp 181-200

https://doi.org/10.36561/ING.17.8

ISSN 2301-1092 • ISSN (en línea) 2301-1106 


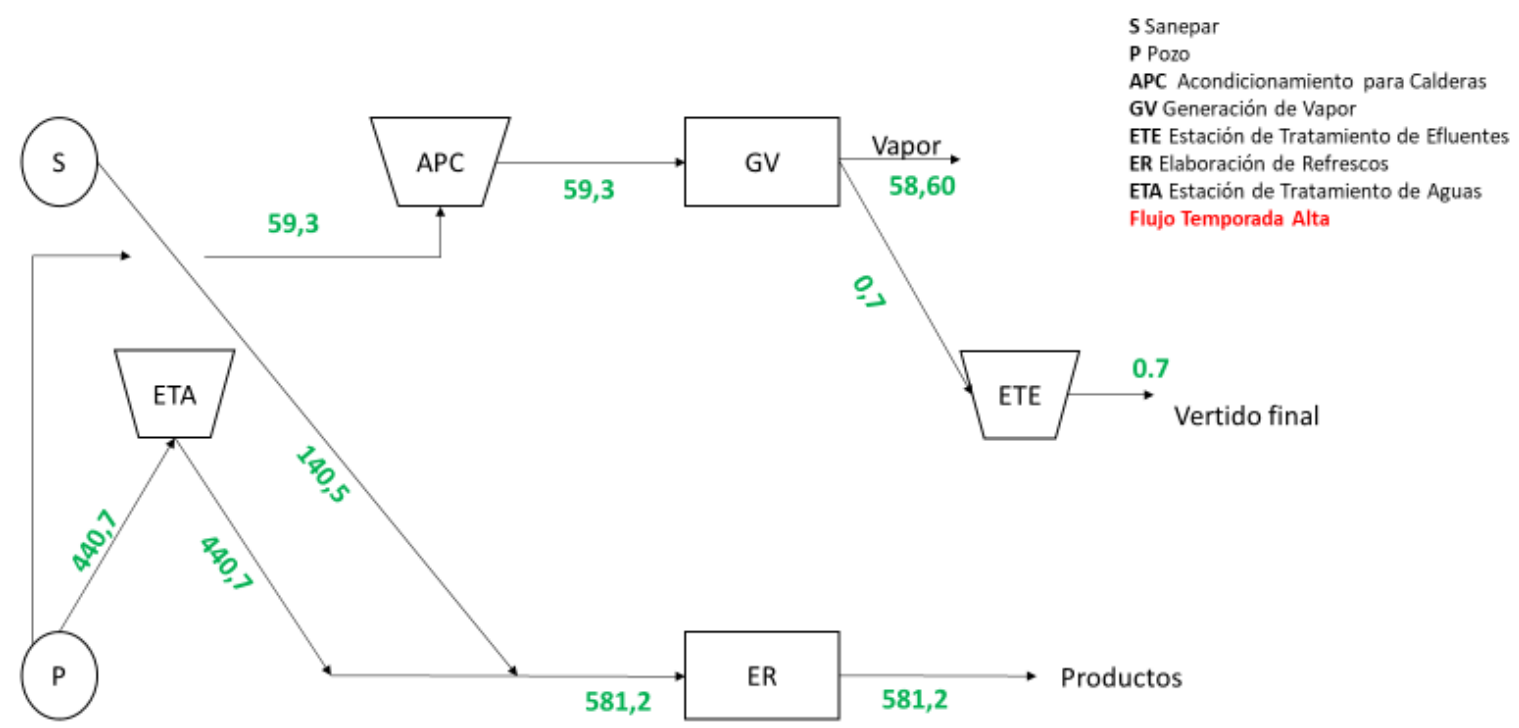

Flujo $\mathrm{j}=$ red industrial $=140,5 \mathrm{~m} 3 / \mathrm{dia}$

$\mathrm{j}=$ poso artesanal $=500 \mathrm{~m} 3 / \mathrm{dí}$

Figura V: Distribución de agua dentro de la planta (m3/día). Temporada Alta. Fuente: Resultado Iteración $K=2$

En la tabla XIII se presenta la comparación de resultados al introducir del problema original NLP directamente en el solucionador MINOS ${ }^{\circledR}$

\begin{tabular}{c|c|c}
\hline Algoritmo & Temporada & Tipo de solución \\
\hline Tradicional en MINOS para NLP & baja: & Óptimo local \\
-0.99 y 1.42 seg. & 308,4108 & No factible \\
& alta: & \\
\hline Floudas et al. Descomposición & 374,021 & Óptimo global \\
GBD en BDM-LP & baja: & Óptimo global \\
0.077 y 0.082 seg & 306,21543 & alta: \\
\hline
\end{tabular}

Tabla XIII: Comparación de resultados de costos totales (USD/día) con dos algoritmos de resolución.

Aplicando el algoritmo propuesto por Floudas et, al, se obtuvo una respuesta más confiable -óptimo global-, que la que se pudo alcanzar con el algoritmo tradicional NLP del solucionador MINOS ${ }^{\circledR}$-óptimo local en el mejor de los casos-; y una velocidad de convergencia notablemente mejor. Este resultado coincide con lo reportado por los autores citados al inicio del trabajo, La rapidez de la convergencia permite la realización de varias corridas y un análisis de sensibilidad cuyos resultados se analizarán en estudio posteriores.

El hecho de que la respuesta alcanzada sea un óptimo global garantiza que, para ese conjunto de datos, la distribución de los recursos hídricos derivada de la solución de las variables de decisión, es la correspondiente al menor costo operativo de la temporada.

Memoria Investigaciones en Ingeniería, 2019. N$^{\circ} 17(1) . p p$ 181-200

https://doi.org/10.36561/ING.17.8

ISSN 2301-1092•ISSN (en línea) 2301-1106 
Más allá de las características estructurales del modelo, es importante analizar ciertos aspectos relacionados al propio resultado práctico de la distribución obtenida y minimización de costos.

La diferencia estructural entre los diagramas de las dos temporadas está en la reutilización del efluente tratado en la unidad de Generación de Vapor. Estas unidades fueron estudiadas como cajas negras, en tal sentido sería ilustrador incorporar un análisis más detallado de lo que está ocurriendo en cada una para proponer concisamente qué forma tomaría esta distribución y si es factible o no. Para evidenciar el alcance del potencial de reducción de costos se muestra en la tabla XIV su variación con base a la situación actual.

\begin{tabular}{l|l|c}
\hline \multirow{2}{*}{ Costo de distribución actual (USD/mes) } & Temporada baja & 8.017 \\
\cline { 2 - 3 } & Temporada alta & $11.890,27$ \\
\hline \multirow{2}{*}{ Costo de distribución óptimo (USD/mes) } & Temporada baja & 7961,72 \\
\cline { 2 - 3 } & Temporada alta & 9797,62 \\
\hline \multirow{2}{*}{ Reducción de costos $(\%)$} & Temporada baja & $0.7 \%$ \\
\cline { 2 - 3 } & Temporada alta & $17.5 \%$ \\
\hline
\end{tabular}

Tabla XIV: Reducción de costos por optimización en la distribución de agua

\section{Conclusiones}

El algoritmo propuesto por Floudas et al., [14], [15] basado en GBD [16] provee una respuesta más confiable -óptimo global- que aquella obtenida del algoritmo tradicional para problemas NLP -óptimo local en el mejor de los casos- y una velocidad de convergencia sensiblemente menor.

Hay sin embargo algunos aspectos del comportamiento modelo, que deberían tomarse en consideración para mejoras y sistematizaciones. Es importante por ejemplo considerar el impacto que pueda tener la diferencia de magnitud entre los coeficientes de la función objetivo (costos) y los coeficientes de algunas restricciones (concentraciones de contaminantes). Los primeros son hasta 100.000 veces mayores que los últimos y como multiplican los valores de los flujos cuyos requerimientos varían entre temporadas, el modelo pierde sensibilidad para el valor del costo de las aguas y se presenta muy sensible a las variaciones estacionales. Por ello se ve que, salvo la recirculación de agua para las calderas, la estructura optimizada en relación al costo es prácticamente la misma para ambas estaciones, lo que facilita su implementación ya que los valores de los flujos no superaron los márgenes operativos de las tuberías y bombas instaladas. Otro elemento para la mejora es el modelaje interno de las unidades. ya que éstas fueron modeladas como cajas negras sin diferenciar las sub-unidades en las que podría aún optimizarse la distribución de agua.

Es evidente el impacto mayor del potencial de reducción de costos durante la temporada alta, cuando más agua se utiliza. Si el incremento en el uso del agua en la temporada alta en relación a la temporada baja coincidiese exactamente con los requerimientos por el aumento de producción -valores constantes para cada temporada- entonces la reducción de costos en ambas temporadas debería haberse mantenido igual, ya que esta reducción de los costos

\footnotetext{
${ }^{77}$ El costo incluye la multa mensual mínima de 1000 USD por violación del límite máximo de captación de agua de pozo. En el caso de la distribución actual de los flujos de agua, esta multa vale para ambas temporadas y en el caso de la distribución óptima, la misma sólo considera la temporada alta.
}

Memoria Investigaciones en Ingeniería, 2019. $\mathrm{N}^{\circ} 17(1)$. pp 181-200

https://doi.org/10.36561/ING.17.8

ISSN 2301-1092 • ISSN (en línea) 2301-1106 
respondería exclusivamente a la optimización de los flujos de agua restantes de los utilizados para la producción directamente. Sin embargo, el aumento de la reducción de costos en lata temporada podría estar indicando ineficiencia en el consumo actual de las aguas en la alta temporada y se abre una oportunidad para optimizar su distribución

\section{Referencias}

[1] Congreso Nacional de la República Paraguaya (2007). Ley No 3239 de los recursos hídricos de Paraguay. Disponible en http://www.bacn.gov.py/leyes-paraguayas/2724/de-los-recursoshidricos-del-paraguay

[2] Senado y cámara de diputados de la provincia de Buenos Aires (1999). Ley 12.257 Código de aguas. Disponible en:

http://www.oas.org/usde/environmentlaw/waterlaw/documents/Argentina-

Codigo_de_Aguas_[Beunos_Aires]_(1999).pdf

[3] Governo do estado de Paraná. (1999). Lei 12726, Política estadual de recursos hídricos e outras providências. Disponible en: https://www.legisweb.com.br/legislacao/?id=241036

[4] Senado y câmara de representantes de la República Oriental del Uruguay. (2009). Ley 18610. Política Nacional de Aguas. Disponible en: http://www.ose.com.uy/descargas/documentos/leyes/ley_18_610.pdf.

[5] N. Franqueiro y J. Alburquerque. Prospecção de ações recomendadas para a gestão estratégica de aguas subterrâneas. Anais XX Congresso Brasileiro de Águas subterrâneas, 2018. Doi: https://doi.org/10.14295/ras.v0i0.29310

[6] A. da C. Reboucas, "Água subterrânea, fator de competitividade," en Anales XIII Congresso Brasileiro de águas subterrâneas. RELOC - Rede Latinoamericana de Organizações de Bacia, 2000.

[7] F. Wilkendorf, Antonio Espuña, y Luis Puigjaner, "Minimization of the Annual Cost for Complete Utility Systems," Chemical Engineering Research and Design, vol. 76, pp. 239-245, 1998. Doi: https://doi.org/10.1205/026387698524866

[8] J. Caballero y I. Grossmann, “Una revisión en el estado del arte en optimización,” Revista Iberoaméricana de automática e Informática Industrial, vol. 4, n 1, pp. 5-23, 2007.

[9] I. Grossmann, J. Caballero, y H. Yeomans, "Mathematical programming approaches to the synthesis of chemical process systems," Korean J. Chem. Eng., vol. 16, pp. 407-426, 1999. Doi: https://doi.org/10.1007/BF02698263

[10] C. Demirhan, W. Tso, G. Ogumerem, y E. Pistikopoulos, "Energy systems engineering," BMC Chemical Engineering, vol. 11, n 1, pp. 2-19, 2019. Doi: https://doi.org/10.1186/s42480-0190009-5

[11] T. Edgar y E. Pistikopoulos, "Smart manufacturing and energy systems," Computers and Chemical Engineering, vol. 114, pp. 130-144, 2018. Doi:

https://doi.org/10.1016/j.compchemeng.2017.10.027

[12] L. Levente, N. Osterwalder, U. Fischer y K. Hungerbühler, "Systematic Retrofit Method for Chemical Batch Processes Using Indicators, Heuristics, and Process Models," Industrial \& Engineering Chemistry Research, vol. 47, $\mathrm{n}^{\circ}$ 1, pp. 66-80, 2008. Doi: https://doi.org/10.1021/ie070044h.

[13] A. Alva-Argaez, y A. Joule, "Optimal design of distributed effluent treatment system in steam assisted gravity drainage oil sand operation," Journal of Cleaner Production, vol. 149, pp. 12331248, 2017. Doi: https://doi.org/10.1016/j.jclepro.2017.02.131

Memoria Investigaciones en Ingeniería, 2019. №17(1). pp 181-200

https://doi.org/10.36561/ING.17.8

ISSN 2301-1092 • ISSN (en línea) 2301-1106 
[14] A. Floudas, y R. Ciric, R., "Strategies for overcoming uncertainties in the heat exchangers network synthesis", Computers Chemical Engineering, vol. 13, nº 10, pp. 1133-1152, 1989.

[15] A. Floudas, M. Tan y J. Broach, "A novel clustering approach and prediction of optimal number of clusters: global optimum search with enhanced positioning," Journal of Global Optimization, vol. 39, nº 2, pp. 323-346, 2007. https://doi.org/10.1007/s10898-007-9140-6

[16] A. M. Geofrion, "Generalized Benders Decomposition," Journal of Optimization Theory and Applications, vol. 10, $\mathrm{n}^{\circ}$ 4, pp. 237-260, 1972.

[17] SANEPAR, Tabela de evolução tarifária, 2018. Disponible en: http://site.sanepar.com.br/sites/site.sanepar.com.br/files/clientes2012/tabeladeevolucaotarifar ia2018.pdf

[18] SUDERHSA, Plano diretor de drenagem para a Bacia do Rio Iguaçu na Região Metropolitana de Curitiba. Curitiba: Instituto das Águas do Paraná, 2002. Disponible en: http://www.aguasparana.pr.gov.br/arquivos/File/pddrenagem/volume3/SUD0103_WR102_FI.p df

[19] SANEPAR, Manual de Projeto de Saneamento. Modulo 1, 2018. Disponible en: https://site.sanepar.com.br/sites/site.sanepar.com.br/files/informacoes-tecnicas/mps-versao2018/modulo_01_-_prescricoes_para_elaboracao_e_apresentacao_de_etp.pdf

[20] B. Murtagh, y M. Saunders, Minos 5.51 user's guide. Standford: Standford University, 2003.

[21] M. Alhaider, L. Fan, y Z. Miao, "Benders Decomposition for stochastic programming-based PV/Battery/HVAC planning," 2016 IEEE Power and Energy Society General Meeting (PESGM), Boston, MA, 2016. Doi: https://doi.org/10.1109/PESGM.2016.7741775

[22] R. Jamalzadeh, F. Zhang, y M. Hong, "An economic dispatch algorithm incorporating voltage management for active distribution systems using generalized benders decomposition," 2016 IEEE Power and Energy Society General Meeting (PESGM), Boston, MA, 2016. Doi: https://doi.org/10.1109/PESGM.2016.7741977

[23] A. Diniz y T. Santos, "Feasibility and optimality cuts for the multi-stage benders decomposition approach: Application to the network constrained hydrothermal scheduling," 2009 IEEE Power \& Energy Society General Meeting, Calgary, AB, 2009. Doi: https://doi.org/10.1109/PES.2009.5275442

[24] W. Yeh, "Optimization methods for groundwater modeling and management," Hydrogeology Journal, vol. 23, pp. 1051-1065, 2015. Doi: https://doi.org/10.1007/s10040-015-1260-3 